\title{
Conceptus-mediated endometrial vascular changes during early pregnancy in mares: an anatomic, histomorphometric, and vascular endothelial growth factor receptor system immunolocalization and gene expression study
}

\author{
L A Silva ${ }^{1,2}$, C Klein ${ }^{3}$, A D Ealy ${ }^{1}$ and D C Sharp ${ }^{1}$ \\ ${ }^{1}$ Department of Animal Sciences, University of Florida, 450 Shealy Drive, Gainesville, Florida 32611, USA, \\ ${ }^{2}$ Department of Animal Sciences, FZEA, University of Sao Paulo, Pirassununga, Sao Paulo 13635-900, Brazil and \\ ${ }^{3}$ Department of Veterinary Science, University of Kentucky, Lexington, Kentucky 40546, USA
}

Correspondence should be addressed to D C Sharp; Email: sharp@ufl.edu

\begin{abstract}
This work examined how the conceptus modulates endometrial tissue remodeling and vascular development prior to implantation in mares. A macroscopic uterine examination was completed at day 21 of pregnancy. In situ morphology revealed that the endometrium involved in encroachment is restricted to the dorsal endometrium immediately overlying the yolk sac. The amount of stromal area occupied by blood vessels and the number of endometrial glands were increased during early pregnancy. Endometrial histomorphometry as well as the endometrial mRNA abundance and immunolocalization of VEGF, VEGFR1, VEGFR2, and Ki-67 was completed at days 14 and 21 of pregnancy, at day 10 of the estrous cycle, and during estrus. No obvious differences in VEGF and VEGFR1 protein localization were detected between pregnant and cycling mares but differential staining pattern for VEGFR2 and Ki-67 was observed. VEGFR2 localized to luminal and glandular epithelium of pregnant mares, while luminal epithelium was negative in cycling mares. Ki-67 staining was weak during the luteal phase but exhibited prominent luminal epithelium staining during estrus. In pregnant mares, all endometrial layers were Ki-67 positive. Quantitative RT-PCR revealed a greater abundance of VEGF mRNA during pregnancy. VEGFR2 transcript abundance was greatest in pregnant mares on day 21 . This study supports the concept that the conceptus plays an active role in directing vasculogenesis within the uterus and thereby establishing hemotrophic nutrition that supports pregnancy after implantation.

Reproduction (2011) 142 593-603
\end{abstract}

\section{Introduction}

Vascular development and remodeling in both maternal and fetal compartments are essential during pregnancy in mammals (Reynolds et al. 2006, Torry et al. 2007). Prior to implantation, the endometrium provides histotroph to support embryo survival and development, but fetal growth increases the demand for gaseous and nutrient exchange (hemotroph) culminating in the fully developed placenta (Burton et al. 2007). Maternoplacental vasculature remodeling parallels the increase in fetal transport of nutrients, respiratory gases, and wastes (Faber \& Thornburg 1983, Reynolds et al. 2006, Torry et al. 2007). Endometrial vascular development and endometrial thickness have been associated with successful implantation in women (Torry et al. 2007). In addition, the endometrial glands have also been suggested as a source of nutrients, growth factors, and cytokines during the entire first trimester of pregnancy in women (Burton et al. 2002, 2007). This conversion from primarily histotrophic nutrition to hemotrophic nutrition is of interest especially in animals with non-invasive epitheliochorial placentae where histotroph is thought to be the major source of nutrition early on (Amoroso 1952), but the mechanisms regulating tissue remodeling, including the dramatic changes in both fetal and maternal vasculature, are not well understood.

Equids present characteristics during early pregnancy that favor their use as an in vivo experimental model for studying the changes in fetal and maternal vascularization. The embryonic vesicle enters the uterus around day 6 after fertilization and is detectable by ultrasound examination starting as early as day 9 (Betteridge et al. 1982, Ginther 1995). The embryonic vesicle is spherical as a result of its glycoprotein capsule, which begins forming in the oviduct, and remains a conspicuous structure until day 25 or so of pregnancy (Chu et al. 1997). The spherical embryonic vesicle exhibits remarkable mobility, traveling throughout the uterine horns and body many times daily, from the earliest time it can be detected until approximately day 15-16 of pregnancy 
(Leith \& Ginther 1984). The mobility phase of the equine embryonic vesicle is well established as an important event in the maintenance of luteal function (McDowell et al. 1988). After day 15, conceptus mobility ceases as it becomes fixed in the posterior segment of one of the uterine horns (Ginther 1983). In this fixed position, the conceptus continues its spherical growth but spreads rostrally and caudally due to relatively low resistance at these areas of the uterus. Studies using Doppler ultrasonography have described significant uterine and embryonic vascular changes at this time in mares (Silva et al. 2005, Ginther \& Silva 2006) as well as cows (Silva \& Ginther 2010). Pertinent to this study, an increase in endometrial blood flow is associated with conceptus presence during the mobility phase of early pregnancy in mares (Silva et al. 2005, Ginther \& Silva 2006). Transient changes in endometrial vascular perfusion accompanied the embryonic vesicle as the vesicle changed location during embryo mobility. However, on day 13, continued presence of the vesicle in the same horn for an average of 7 min stimulated an increase in vascularity of the endometrium (Silva et al. 2005). Furthermore, endometrial blood flow was shown to increase markedly after fixation (Silva et al. 2005, Silva \& Ginther 2006).

Not coincidentally, shortly after the equine embryonic vesicle becomes fixed, encroachment of the dorsal endometrium on the embryonic vesicle can be observed via ultrasonography (see Fig. 1; Silva \& Ginther 2006). It is not known whether encroachment of the dorsal endometrium on the conceptus reflects tissue hypertrophy or hyperplasia. Nor is it known what regulatory forces are at play to institute such a change. However, we suggest that the endometrial vascular remodeling in mares is modulated by the conceptus, creating a uterine environment that will support further conceptus development, and assuring optimal conditions for the dynamic events of mobility, fixation, maternal recognition of pregnancy, uterine immune modulation, implantation, and eventually placentation.

It is likely that the early equine conceptus alters endometrial vascularity by the production of vascular stimulants. In this regard, estrogens stimulate increased uterine blood flow in the sow, cow, and ewe (Ford et al. 1982a). In vitro studies have shown that day 12 porcine embryos (Ford et al. 1982b) and day 16 bovine embryos (Shemesh et al. 1979, Chenault 1980) produce estrogens. A marked production of estrogen by the equine conceptus occurs as early as day 12 (Zavy et al. 1984, Raeside et al. 2004). Thus, estrogen has vasostimulatory properties, and estrogen production by the conceptus is temporally associated with stimulation of uterine vascularity in cattle, pigs, and horses. The blastocysts of many species also secrete a variety of prostaglandins, and it has been also proposed that conceptus prostaglandins stimulate increased uterine blood flow (Lewis 1989).

There are also critical growth factors involved in the physiological regulation of blood vessel formation and maintenance, and the actions of these molecular players must be very carefully orchestrated in terms of time, space, and concentration so as to form a functioning vascular network (Risau 1997, Yancopoulos et al. 2000, Jain 2003, Zygmunt et al. 2003). The crucial factors regulating angiogenesis and vasculogenesis belong to

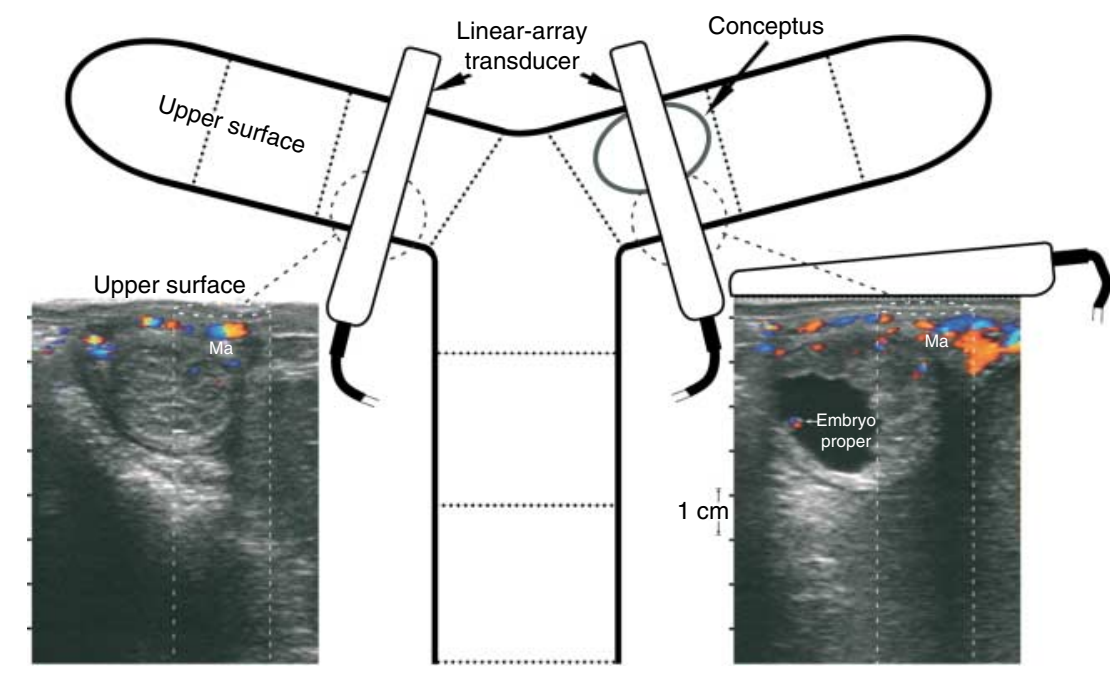

Figure 1 Uterine transrectal ultrasound scanning positioning. A diagram of the uterus with the ultrasonograms obtained from scanning the posterior segment of each uterine horn. The uterine horn on the right contains the fixed conceptus. The dorsal endometrium, which borders the mesometrial attachment area, exhibits disproportionate growth (dorsal encroachment) when compared with the ventral endometrium. More intense blood flow (colored spots) is also observed at this dorsal area endometrium and mesometrium when compared with the opposite uterine horn. The blue and red colors indicate the direction of the blood flow related to the scanning probe plane. The intensity of each color represents flow velocities. The colored spot at the embryo represents the beating heart. The uterine shape in mares permits subjective segmentation into nine segments for study purposes (dashed lines) to precisely determine the locations for data and tissue collection. Ma, mesometrial attachment (adapted from Silva \& Ginther (2006)). 
the endothelial growth factor family. The most important are VEGFA (termed VEGF herein) and its receptors VEGFR1, VEGFR2, and VEGFR3 (Yancopoulos et al. 2000, Ferrara et al. 2003, Ferrara 2004, Roy et al. 2006). Nitric oxide is another factor involved in tissue vascularization by stimulus of vessel dilatation and permeability and decreasing tone. Many studies have shown association of increased gene expression of the major angiogenic factors, as VEGF and eNOS, with altered placental growth and vascular development (Reynolds \& Redmer 2001, Redmer et al. 2005, Reynolds et al. 2005).

Use of an angiogenic inhibitor before or after implantation in mice resulted in resorption of all embryos (Klauber et al. 1997), which supports the hypothesis that angiogenesis is a critical component of normal implantation/placentation in early stages of pregnancy. The nature of the signals responsible for induction and control of endometrial angiogenesis has been the focus of many studies (Girling \& Rogers 2005, Bourlev et al. 2006, Hervé et al. 2006). Estrogen is recognized as one of the driving forces for increased uterine blood flow through both rapid and delayed actions, via binding to its receptors in the uterine artery wall, and especially at the uterine artery endothelium (Heryanto \& Rogers 2002, Mendelsohn 2002, Albrecht et al. 2003).

The main hypothesis of this study is that the conceptus modulates endometrial tissue remodeling and vascular changes prior to implantation in mares. To test that hypothesis, endometrium was obtained from cycling mares and from pregnant mares on days 14 and 21 and examined for potential differences in in situ morphology; histomorphometry; and immunolocalization of VEGF, its receptors, and $\mathrm{Ki}-67$, a proliferation marker; and gene expression quantification of VEGF and its receptors.

\section{Results}

\section{Macroscopic evaluations}

Differential gross and vascular morphology between the two uterine segments, visualized by color-Doppler ultrasonography, have been described in mares in a previous work (Silva \& Ginther 2006; Fig. 1). The in situ macroscopic evaluation of the uterus from four pregnant mares on day 21 revealed remarkable new findings and, by visual examination, confirmed the vascular and anatomic differences found by the previous colorDoppler ultrasonography studies between the posterior segments of the uterine horns ipsilateral and contralateral to the conceptus. Figure 2 shows representative pictures from our macroscopic findings. The intact conceptus with its membranes and primordial vascular system is depicted in Fig. 2A. The incision was made through the dorsal aspect of the uterus. The confluence of the incision edges coincides with the area of the
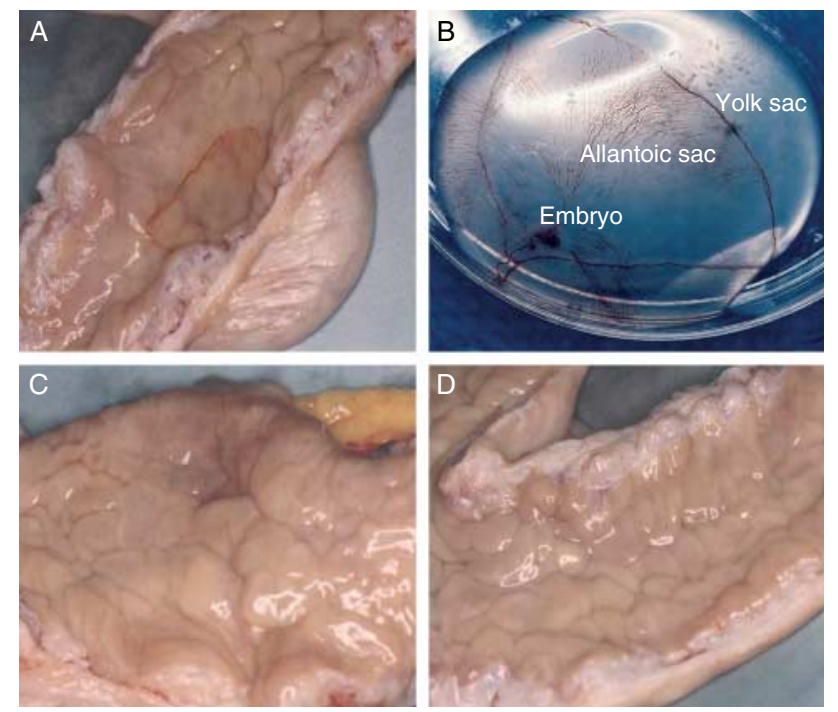

Figure 2 Macroscopic examination of the conceptus and endometrium in the pony mare. The posterior segment of both uterine horns and the conceptus from a mare on day 21 of pregnancy is presented. (A) The posterior segment of the horn ipsilateral to the conceptus and the dorsal area of the endometrium (around the conceptus and adjacent to the mesometrial attachment). (B) Conceptus after its removal. (C) Segment of the horn after removal of the conceptus. (D) Uterine horn from a cyclic mare.

endometrium in intimate contact with the conceptus yolk sac membrane. After separation of the conceptus and endometrium, several conceptus structures were visualized (Fig. 2B). It contains the embryo proper, with intensely vascularized allantoic sac, and the yolk sac. Also, small points on the yolk sac surface, like small sand grains, likely represent patches of adherence between the yolk sac and the endometrium. The exposed endometrium at this region of attachment (Fig. 2C) exhibited more edema and more intense red coloration when compared with the endometrium from the dorsal area of the contralateral uterine horn (Fig. 2D). The differential endometrial morphology observed at the dorsal area of the posterior segment of the ipsilateral horn was observed only in the segment containing the conceptus. The changes observed were strictly localized at the fixation site of the conceptus. Moreover, these changes were observed only in the dorsal area of the endometrium.

\section{Morphometric evaluations}

Analysis of the vascular supply, endometrial gland area, and the number of endometrial glands between uterine horns and among the four reproductive statuses is presented in Table 1. The amount of stromal area occupied by blood vessels was markedly greater in the endometrium of pregnant mares than cyclic mares. When all endometrial data were compared among reproductive statuses, no differences were found 
Table 1 Analysis of the vascular supply, endometrial gland area, and number of endometrial glands between the uterine horns and among four reproductive statuses in mares.

\begin{tabular}{|c|c|c|c|c|c|}
\hline End point ${ }^{a}$ & Pregnant day 21 & Pregnant day 14 & Estrus & Diestrus & $P$ value \\
\hline \multicolumn{6}{|c|}{ Blood vessels ( $\%$ of stroma area) } \\
\hline Contralateral horn & $2.89 \pm 0.56^{*}$ & $2.44 \pm 0.48^{*}$ & $1.68 \pm 0.26^{*,+}$ & $\begin{array}{l}1.25 \pm 0.55 \\
0.52+0.29^{+, \neq}\end{array}$ & $\begin{array}{l}0.0001 \\
0.0023\end{array}$ \\
\hline$P$ value & 0.0001 & 0.1220 & 0.0583 & 0.0950 & \\
\hline All endometrium & $4.43 \pm 0.55^{*}$ & $3.48 \pm 0.68^{*}$ & $1.29 \pm 0.19^{+}$ & $0.93 \pm 0.22^{\dagger}$ & 0.0001 \\
\hline \multicolumn{6}{|c|}{ Glands (\% of total area) } \\
\hline Ipsilateral horn & $48.6 \pm 1.7^{*}$ & $40.0 \pm 2.3^{*,+}$ & $36.1 \pm 1.2^{+}$ & $44.9 \pm 3.2^{*,+}$ & 0.0015 \\
\hline Contralateral horn & $49.0 \pm 1.5^{*}$ & $35.5 \pm 2.1^{\dagger}$ & $38.4 \pm 3.0^{\dagger}$ & $45.8 \pm 1.2^{*}$ & 0.0001 \\
\hline$P$ value & 0.4386 & 0.1351 & 0.2194 & $0 . \overline{3} 721$ & \\
\hline All endometrium & $48.8 \pm 1.3^{*}$ & $38.2 \pm 1.7^{+}$ & $37.1 \pm 1.2^{+}$ & $45.4 \pm 1.2^{*}$ & 0.0001 \\
\hline \multicolumn{6}{|c|}{ Number of uterine glands } \\
\hline Ipsilateral horn & $70.4 \pm 8.7^{*,+}$ & $90.4 \pm 13.8^{*}$ & $47.2 \pm 4.7^{+}$ & $60.6 \pm 4.0^{*,+}$ & 0.0367 \\
\hline Contralateral horn & $72.6 \pm 4.7$ & $83.3 \pm 13.1$ & $53.6 \pm 7.4$ & $57.8 \pm 4.1$ & 0.1052 \\
\hline$P$ value & $0 . \overline{4} 145$ & $0 . \overline{3801}$ & $0 . \overline{2} 338$ & $0 . \overline{3} 236$ & \\
\hline All endometrium & $71.5 \pm 3.9^{*,+}$ & $89.7 \pm 6.6^{*}$ & $50.1 \pm 3.4^{\ddagger}$ & $59.2 \pm 3.0^{\dagger, \neq}$ & 0.0004 \\
\hline
\end{tabular}

Different superscripts indicate statistics differences $(P<0.05)$ among reproductive statuses. Differences between uterine horns are indicated by the respective $P$ values. Bold characters indicate end points with high values in pregnant status.

${ }^{a}$ Values presented are averages calculated based on measurements randomly taken from three microscopic fields at $200 \times$ magnification in each endometrial stratum and both uterine horns. Values for all endometrium are the average of all measurements.

between pregnant mares on days 14 and 21 of pregnancy. However, the ipsilateral horn of pregnant mares on day 21 exhibited greater blood vessel area than the contralateral horn and the ipsilateral horn of pregnant mares on day 14 . The contralateral horns of mares pregnant for 14 and 21 days did not differ. Blood vessel area was not different between cyclic mares in follicular and luteal phases and no difference was found when the ipsilateral horn was compared with the contralateral horn in either follicular or luteal phases (Table 1).

The area of all endometrium occupied by uterine glands was similar between pregnant mares on day 21 and cyclic mares during the luteal phase, but larger than that in pregnant mares on day 14 and cyclic mares during the follicular phase. Area of the endometrium occupied by uterine glands in pregnant mares on day 14 and cyclic mares during the follicular phase did not differ (Table 1). The number of uterine glands per endometrial area was greater in the pregnant groups compared with the cyclic groups. The number of glands did not differ between uterine horns.

Similar analyses were performed comparing the two endometrial strata: the stratum spongiosum (which included the stratum compactum) and the stratum basalis (Fig. 3). In the stratum spongiosum, the superficial area of the endometrium, the area of blood vessels did not differ among reproductive statuses in the contralateral uterine horn (Fig. 3A). However, the blood vessel area was larger in the ipsilateral horn of pregnant mares than cyclic mares. In addition, blood vessel area in the ipsilateral horn was larger than that in the contralateral horn only in pregnant mares on day 21 . In the stratum basalis, the area occupied by blood vessels was in general twofold larger when compared with the stratum spongiosum in any of the horns or pregnancy statuses (Fig. 3B). Both uterine horns in pregnant mares on day 21 exhibited more blood vessels than in the uterine horns
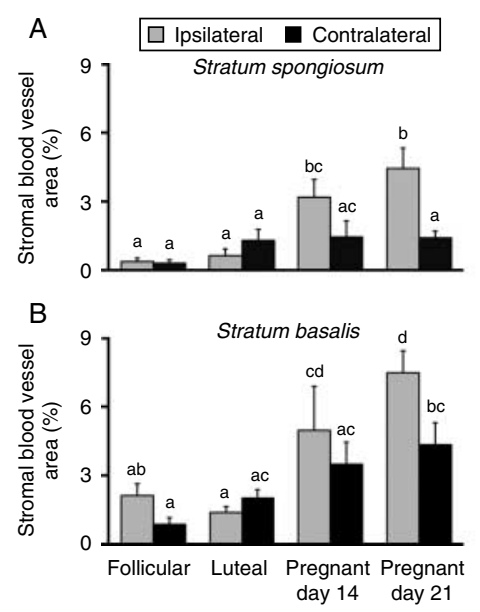

Reproduction (2011) 142 593-603
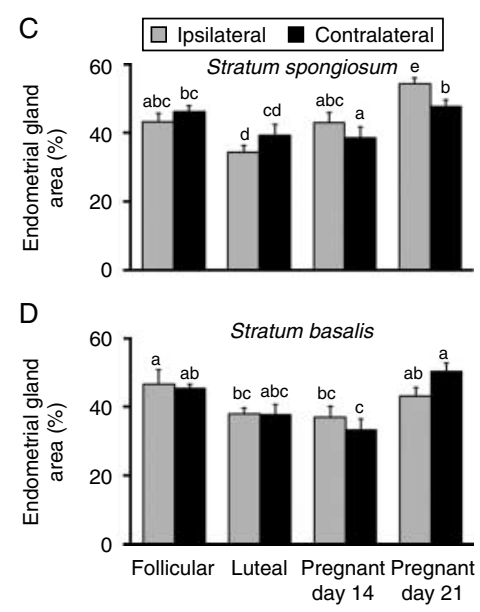

Figure 3 The effect of pregnancy status and stage of pregnancy on stromal blood vessel area and endometrial gland area in pony mares. (A) Percentage of stromal area occupied by blood vessels in the stratum spongiosum. (B) Percentage of stromal area occupied by blood vessels in the stratum basalis. (C) Percentage of stromal area occupied by glands in the stratum spongiosum. (D) Percentage of stromal area occupied by glands in the stratum basalis. Differences based on pregnancy status and horn location are indicated by different superscripts within each graph $(P<0.05)$. 
of cyclic mares. The pregnant mares on day 14 appeared to be transitional between the cyclic state and advanced pregnant state (day 21) relative to increased blood vessels. The blood vessel area in the ipsilateral horn was largest in the pregnant mares on day 21.

The area occupied by endometrial glands in the stratum spongiosum was larger only in the ipsilateral horn of pregnant mares on day 21 when compared with the ipsilateral horn of the other groups or with the contralateral horn in the same group (Fig. 3A). Interestingly, in the stratum basalis, the area occupied by endometrial glands was larger in the contralateral horn of pregnant mares on day 21 than in the pregnant mares on day 14 or during follicular phase but similar to that during luteal phase (Fig. 3B). No differences were observed either between statuses in the blood vessel area in the ipsilateral horn or between horns within statuses.

\section{Immunohistochemical localization studies}

No difference in staining pattern was found for VEGF and VEGFR1 antigens among the studied reproductive statuses (Fig. 4). However, differential staining patterns were found for VEGFR2 (Fig. 5). The anti-VEGFR2 serum stained the luminal and glandular epithelial cells, endothelium, and, less intensely, some of the stromal cells in pregnant mares. In cyclic mares, the luminal epithelium did not stain at all or presented only a few cells with weak staining during the luteal phase. The absence of staining was more pronounced during the follicular phase. Staining of stromal cells was observed although with lower intensity than that in the luminal or glandular epithelial cells.

These endometrial samples were also stained for Ki-67 reactivity (Fig. 6). The anti-Ki-67 serum staining was rarely observed in stromal and glandular epithelial cells during the luteal phase. However, it exhibited prominent staining in the majority of the luminal epithelial cells and in a few stromal cells during follicular phase. In pregnant mares, the staining was present in all endometrial cell layers. Pregnant mares on day 21 exhibited a staining pattern similar to pregnant mares on day 14 . There appeared to be a greater number of positive cells in all endometrial layers at day 21.

\section{Quantitative RT-PCR studies}

The relative abundance of VEGF, VEGFR1, and VEGFR2 mRNA was examined in the endometrium of pregnant and cyclic mares (Fig. 7). Endometrial biopsies were collected from the horn ipsilateral to the conceptus, the preovulatory follicle, or corpus luteum depending on the animal and its pregnancy status. Abundance of $V E G F$ mRNA was greater $(P<0.05)$ in endometria from pregnant ponies than from samples collected during
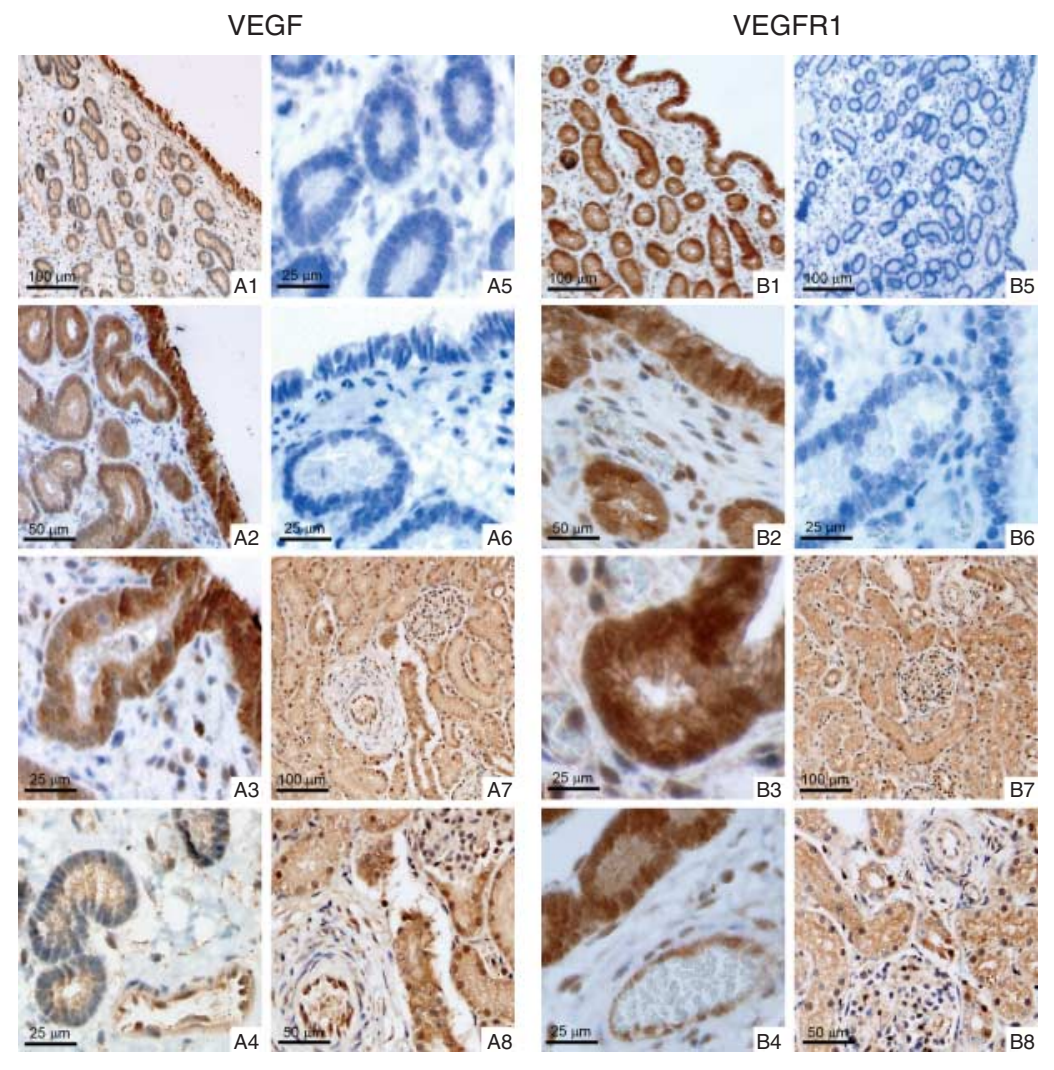

Figure 4 Immunolocalization of VEGF (A) and VEGFR1 (B) in mare endometrium. Sections were stained with anti-VEGF or anti-VEGFR1 serum (1:200 dilution). A representative sample is illustrated at several magnifications. Controls include those lacking primary antisera (A5, A6, B5, and B6) and positive controls from kidney sections (A7, A8, B7, and B8). 


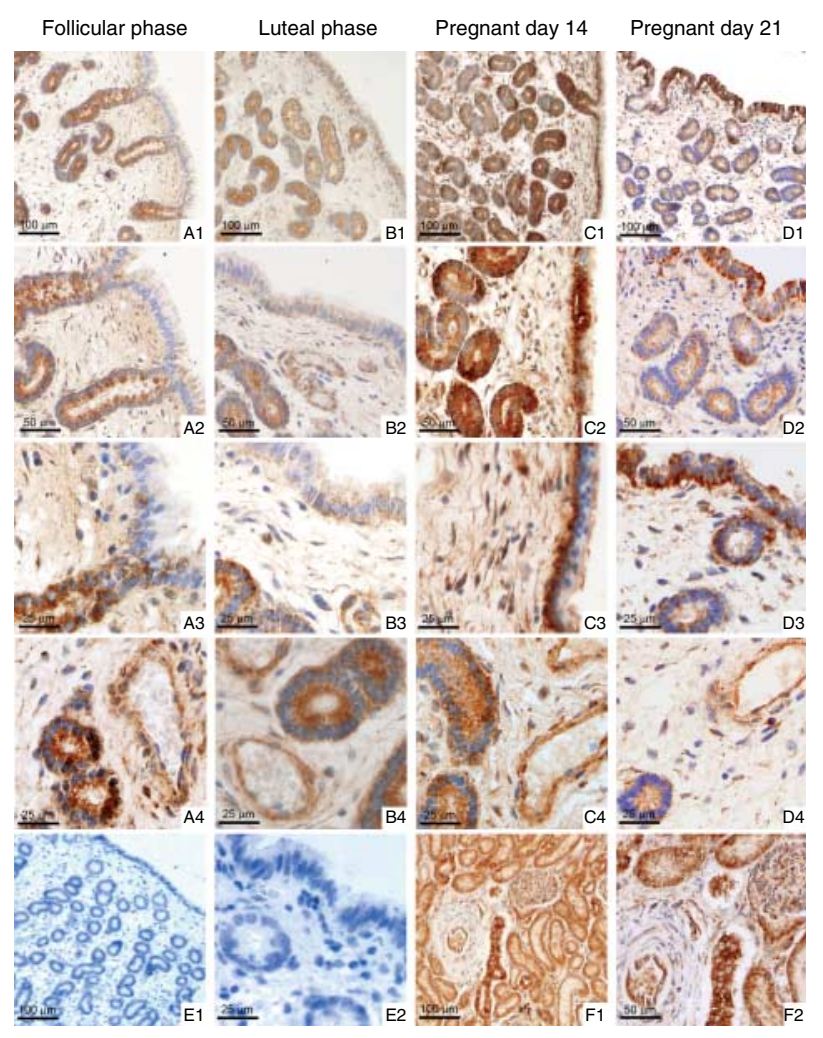

Figure 5 Immunolocalization of VEGFR2 in endometrium from cyclic and pregnant mares. Representative pictures taken at several fields of magnification are presented for endometrial sections from cyclic mare during follicular (A) and luteal (B) phases and pregnant mares at days $14(C)$ and 21 (D) of pregnancy. Sections were stained with a 1:200 dilution of anti-VEGFR2 serum. Negative controls (E) and kidney sections (F) also are presented.

the follicular and luteal phases in cyclic ponies (Fig. 7A). Abundance of VEGFR2 mRNA was greatest $(P<0.05)$ in pregnant mares on day 21 (Fig. 7B) but no differences were detected in VEGFR1 mRNA concentrations across stages (Fig. 7C).

\section{Discussion}

Several yet undescribed features of uterine responses to the equine placenta were uncovered in this work. One key feature is the events surrounding conceptus orientation and fixation of the conceptus onto the dorsal but not ventral endometrium (see Fig. 1). The literature does not instruct as to whether this encroachment reflects tissue hyperplasia, tissue hypertrophy, or both. By day 21 of pregnancy, the spherical embryonic vesicle is fixed in the posterior segment of one of the uterine horns and exhibits dramatic endometrial vascularity and morphology differences between uterine horns observed with color-Doppler ultrasonography (Silva et al. 2005, Silva \& Ginther 2006). These authors reported greater vascularity in the horn ipsilateral to the conceptus at both endometrial and mesometrial levels. The area presenting the greatest vascularity was localized in the endometrium dorsally, bordering the mesometrial attachment, and in immediate contact with the fixed conceptus. Substantial growth of the endometrium in the dorsal area of the fixation site was observed. The present in situ morphological study confirmed by visual inspection the previous color-Doppler ultrasonographic findings. The dorsal endometrium at the fixation site was more edematous (thicker) and hyperemic than any other area of the endometrium. Lefranc \& Allen (2007) described by endoscopy approach a discrete hyperemia at the endometrium in contact with the conceptus between days 20 and 30 of pregnancy. Our findings demarcated the endometrial edematous and hyperemic area. This area was restricted to the dorsal endometrium immediately overlying the conceptus, bordering the mesometrial attachment region, and coincided with the surface of the yolk sac. The observation that dorsal endometrial encroachment was so intimately and discretely associated with conceptus position was not anticipated, despite our hypothesis that the endometrial changes may reflect conceptus influence. Conceptus orientation and alignment of the yolk sac to the dorsal endometrium (Ginther 1983) is important for the correct

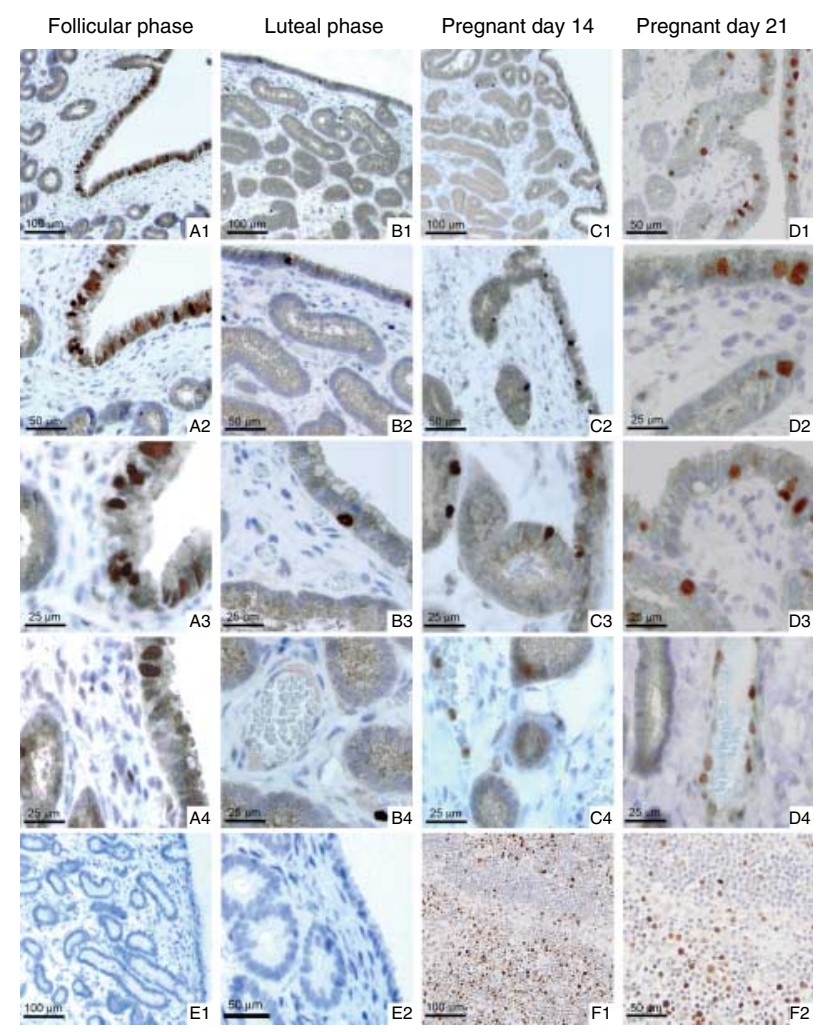

Figure 6 Immunolocalization of $\mathrm{Ki}-67$ in endometrium from cyclic and pregnant mares. Representative pictures taken at several fields of magnification are presented for endometrial sections from cyclic mare during follicular (A) and luteal (B) phases and pregnant mares at days 14 (C) and 21 (D) of pregnancy. Sections were stained with a 1:200 dilution of anti-Ki-67 serum. Negative control (E) and kidney sections for positive control (F) are also presented. 


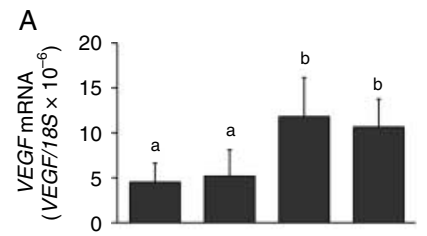

$\mathrm{B}$
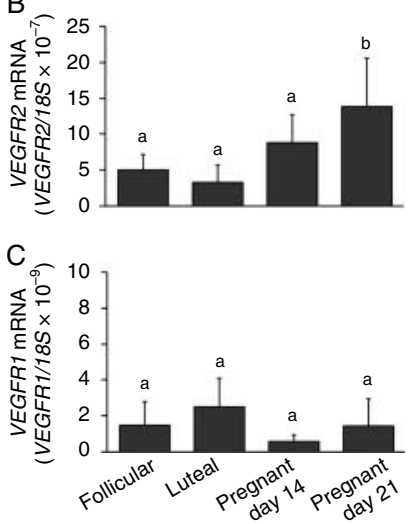

Figure 7 Effect of the reproductive status on the relative abundance of VEGF, VEGFR2, and VEGFR1 mRNA in mare endometrium. Endometrial biopsies were taken from the posterior segment of the uterine horn ipsilateral to the conceptus or to the side of the largest follicle or corpus luteum in cyclic mares. Abundance of mRNA relative to an internal control (18S) is presented for VEGF (Panel A), VEGFR2 (Panel B), and VEGFR1 (Panel C). Stage-dependent differences in mRNA abundance $(P<0.05)$ are indicated within each graph by different superscripts.

positioning of the umbilical cord at the area of the mesometrial attachment around day 40 of pregnancy (Ginther \& Silva 2006, Silva \& Ginther 2006). Furthermore, the physical interaction of dorsal endometrial encroachment with uterine smooth muscular contraction, along with conceptus architecture, may work together to effect conceptus rotation to an appropriate position for eventual umbilical cord attachment. Our current observations suggest that conceptus factors may play an active role in stimulating changes to the dorsal endometrium leading to encroachment.

Using color-Doppler ultrasonography, it has been shown that this process of orientation occurs immediately after fixation and no further changes in embryonic vesicle positioning (i.e. rotation) occur (Silva \& Ginther 2006). In addition, it has been shown that disorientated embryonic vesicles are frequently associated with lack of dorsal endometrial encroachment, poor endometrial vascularity, and embryonic loss (Ginther \& Silva 2006). These studies provide support for the notion that the correct positioning of the embryonic vesicle is crucial for its survival and development and suggest an active role by the conceptus.

The morphometry studies indicated increased vascular and glandular activity in dorsal endometrium ipsilateral to the conceptus compared with dorsal endometrium contralateral to the conceptus. Not surprisingly, mares that were pregnant tended to exhibit greater area of the endometrium occupied by blood vessels compared with nonpregnant mares, and mares at 21 days of pregnancy exhibited the greatest area of endometrium occupied by blood vessels. The increase in dorsal endometrial blood vessels supports observations of increased blood flow with color-Doppler ultrasonography and may also partially explain dorsal endometrial encroachment, as the area occupied by blood vessels was 2- to 3.5-fold higher in the stratum basalis and four- to eight-fold higher in the stratum spongiosum in pregnant mares. Furthermore, blood vessel area in pregnant mares on day 21 was greater in the uterine segment occupied by the conceptus, suggesting intense localized endometrial stimulation.

Dorsal endometrial encroachment may also reflect an increase in the area of endometrium occupied by endometrial glands, although this increase was of a lesser magnitude than that of blood vessels, and was most prominent only in the endometrium ipsilateral to the conceptus. Given the hypothesis that the conceptus plays an active role in the changes to the dorsal endometrium, it seems reasonable that the major increase in endometrial glands occurs adjacent to the conceptus.

In that regard, during the dissection for in situ morphology observations, we noticed some adherence of the yolk sac to the endometrium. There appeared to be multiple small patches of contact between the yolk sac surface and the endometrium, which are candidates for future studies of tissue adhesion molecules. The potential function of such adhesion points is not known but seems a reasonable part of the mechanisms aimed at directing conceptus orientation for both histotrophic and hemotrophic nutrition sources.

We propose that conceptus nourishment before umbilical cord formation is provided predominately by uterine glands at the dorsal endometrial area. The increased number of blood vessels and uterine glands at this area of the endometrium in intimate contact with the thinner bilaminar layer favors delivery of nutrients and potentially other embryonic-maternal communications.

VEGF and VEGFR1 exhibit similar staining patterns in the endometrium of all four reproductive statuses. Intense VEGF reaction was observed in the luminal epithelium. Glandular epithelial cells and endothelial cells were also stained but not as strong as the luminal epithelium. Some stromal cells were also stained, although they were dispersed in the stroma with less intense staining when compared with the other endometrial cell layer. This differential stromal cells staining suggests specificity of the immunolabeling. Our results for VEGF and VEGFR1 staining differ from a recent immunohistochemistry work done in mares (Allen et al. 2007). In previous work, no staining for VEGF and weak staining for VEGFR1 at the luminal epithelium and 
stroma was observed during estrus and no staining was detected for VEGF in the stroma nor for VEGFR1 at stroma and luminal epithelium during diestrus. In endothelium, VEGF staining was weak and VEGFR1 staining was essentially absent during estrus while the opposite was observed during diestrus.

The staining pattern presented by VEGFR2 in our study differed between pregnant and cyclic mares. VEGFR2 staining was absent or weak at the luminal epithelial cells in cyclic mares. We found VEGFR2 in the endothelium, luminal and glandular epithelium, and in some of the stromal cells. This stromal cell-labeling pattern, as observed for VEGF and VEGFR1, suggests specificity of our VEGFR2 labeling system, as opposed to nonspecific staining of all cell types. Allen et al. (2007) did not find VEGFR2 staining in the luminal epithelium of mares during diestrus or early pregnancy, but a weak stain during estrus. In our study, during estrus, the luminal epithelial cells did not stain positive for VEGFR2. Kaczmarek et al. (2008) in an immunostaining study on sows reported a staining pattern similar to our findings for VEGF and its receptor system during the estrous cycle and early pregnancy. Upregulation of VEGFR2 expression has been correlated with increased levels of estrogens and VEGF (Hervé et al. 2006) and the early equine conceptus produces prodigious amounts of estrogens at the luminal epithelium surface (Zavy et al. 1984). The link between estrogens and VEGF production has been demonstrated (Cullinan-Bove \& Koos 1993, Bausero et al. 1998). Rockwell et al. (2002) showed that immunoneutralization of VEGF reduced the uterine edema induced by estrogens and blocked implantation. VEGF acts primarily through binding to VEGFR2 (Ferrara et al. 2003, Ferrara 2004); however, estrogens also indirectly induce VEGFR2 expression via VEGF production (Hervé et al. 2006). This suggests that the strong VEGFR2 expression found at the luminal epithelium in pregnant mares could reflect conceptus-produced estrogens. The strong staining of VEGFR2, at the luminal epithelium of pregnant mares, but not found in the luminal epithelium of cyclic mares, likely indicates a pivotal event in the endometrial vascular changes prior to implantation.

Ki-67 immunohistochemistry served as a marker for cell proliferation and, as might be expected, was prominent in luminal epithelium during estrus, but not diestrus. In pregnant mares, however, Ki-67 staining was present in all endometrial cell layers as luminal epithelium, glandular epithelium, stroma, and endothelium. An interesting inverse relationship between Ki-67 and VEGFR2 was observed in luminal epithelium of mares during estrus. Whereas VEGFR2 was evident in glandular epithelium, staining appeared to cease abruptly at the mouth of endometrial glands at the border between glandular and luminal epithelium. $\mathrm{Ki}-67$, on the other hand, was observed only in luminal epithelium of estrous mares and did not appear to localize in the glandular epithelium. It is interesting to contemplate whether the tissue remodeling needs of estrus and pregnancy, as reflected by these immunohistochemistry data, are qualitatively different or simply quantitatively different.

Finally, VEGF and VEGFR2 mRNA expressions were elevated in pregnant mares when compared with cyclic mares, likely reflecting conceptus estrogens (CullinanBove \& Koos 1993, Bausero et al. 1998, Rockwell et al. 2002) and the consequent VEGF-induced expression of VEGFR2 (Hervé et al. 2006). The expression of VEGFR2 in pregnant mares on day 21 was greater than that on day 14. Based on immunolocalization staining, this receptor was more intense at the luminal and glandular epithelium when compared with the stroma. This could reflect VEGF release from the conceptus and/or repopulation of the estrogen receptors in the endometrium. The levels of VEGFR1 mRNA did not differ among the reproductive statuses and its detection was only possible after more than 30 cycles of amplification, suggesting very low expression in all reproductive statuses studied.

Our main hypothesis that endometrial angiogenesis and architecture changes are mediated by the presence of the conceptus was supported. The events of early pregnancy in mares such as conceptus mobility phase, fixation, orientation, and dorsal encroachment of the endometrium on the conceptus side are temporally orchestrated to remodel the maternal uterine vascular system for the ultimate purpose of providing hemotrophic nutrition. Our findings suggest local stimulation of the endometrial vascular system by the conceptus. However, these results also indicate that endometrial architecture is modulated by local and systemic forces during pregnancy. A notable finding is that production and localization of VEGFR2 and proliferative cells (Ki-67) are diametrically differentially regulated. Preliminary real-time PCR results suggest that the high level of expression of VEGF and VEGFR2 in the pregnant mares could account for the observed increase in vascular architecture during pregnancy.

\section{Materials and Methods}

\section{Animals}

Animals were handled in accordance and with the approval of the University of Florida Institutional Animal Care and Use Committee. Pony mares aged 4-16 years were housed at the University of Florida Horse Unit in Lowell, FL, and maintained on pasture with free access to grass, water, and trace-mineralized salt and fed supplemental grass hay (Coastal Bermudagrass) as needed to maintain body condition around 5.0 throughout the experiments (body condition scale from 1 to 9; Henneke et al. 1983). Mares were teased daily to evaluate reproductive behavior and the reproductive tract was palpated transrectally every third day unless an ovarian follicle $\geq 25 \mathrm{~mm}$ was detected, in which case mares were palpated daily. 
Mares to be bred were mated to a stallion of known fertility or inseminated with fresh semen from the same stallion daily, beginning at first detection of an ovarian follicle $\geq 30 \mathrm{~mm}$ until ovulation was detected.

For the first study (in situ macroscopic evaluation), four day 21 pregnant ponies were killed for collection of the entire reproductive tract. Tissues derived from these studies were harvested and preserved for future studies of angiogenic and proliferative factors. The mares were bred using the protocol described above. For killing, pre-sedation with a xylazine $(0.5 \mathrm{mg} / \mathrm{lb}$ i.v.) was administered, after which an overdose of pentobarbital sodium and $50 \mathrm{mg}$ phenytoin sodium was administered ( $1 \mathrm{ml} / 10 \mathrm{lbs}$ bw i.v.; Beuthanasia-D Special; Schering-Plough Animal Health, Summit, NJ, USA). After confirmation of death (absence of breathing, heartbeat, and corneal reflex), the reproductive tract was surgically removed, examined macroscopically, and tissues were collected.

Endometrial samples from day $14(n=5)$ and day $21(n=11)$ pregnant mares and cyclic mares during follicular $(n=6)$ and luteal $(n=6)$ phases were collected by transcervical endometrial biopsy. Pony mares were randomly assigned to be bred (day 14 or 21), or cyclic (follicular (estrus) or luteal (day 10 after estrus) phase of the reproductive cycle), and were scanned daily by ultrasound to monitor the size of the largest follicle and uterine characteristics (Ginther 1995). Mares assigned to the two pregnant groups were either inseminated artificially with raw semen collected from a donor pony stallion with known fertility or bred naturally to the same stallion every day after first detection of a dominant follicle $(>35 \mathrm{~mm})$ until ovulation was confirmed. Pregnancy diagnoses were done on days 10-12 after ovulation. Pregnant mares were used for endometrial biopsies on days 14 and 21 and nonpregnant mares were biopsied during the follicular phase and during the luteal phase of the reproductive cycle. The follicular phase was defined as having a follicle $\geq 30-35 \mathrm{~mm}$ of diameter, uterine echotexture equal or larger than 3.5 (1-4; Ginther 1995), and evidence of estrous behavior. The luteal phase was defined as nonbred mares on day 10 after ovulation, presence of a corpus luteum, and no estrous behavior (Ginther 1992).

\section{Endometrial sampling}

Endometrial tissue biopsies were taken from the dorsal area of the posterior segment of each uterine horn from all mares. The uterus of mares has a T- or Y-shaped resting on the abdominal organs (Ginther 1992) and due to that easily permits evaluating a subjective spatial segmentation of the uterus, by palpation or ultrasound scanning, in nine symmetrical segments, three in each horn and three in the body, as used for embryonic vesicle mobility trial studies (Ginther 1984; see Fig. 1). All mares were sedated (200-300 mg xylazine hydrochloride; i.v.) and a basket-type equine uterine biopsy forcep was used transvaginally for sampling the endometrium. Ultrasound scanning was performed immediately before biopsies to evaluate the endometrium dorsal encroachment at the posterior segment of the uterine horns and also after the biopsies to confirm the correct location of sampling. The endometrial tissue was sectioned into two parts. One of the sections was fixed by immersion in $10 \%$ [v:v] buffered formal saline for $24 \mathrm{~h}$ at $4{ }^{\circ} \mathrm{C}$ before processing for histology. The second section was placed in a plastic tube and snap frozen in liquid nitrogen and stored at $-80{ }^{\circ} \mathrm{C}$ for subsequent RNA extraction. After the $24 \mathrm{~h}$ fixation period, the endometrial samples were trimmed, dehydrated, and embedded in paraffin wax. The paraffin blocks were then cut in sections of $4 \mu \mathrm{m}$ and the layers placed onto poly-L-lysinecoated glass slides. Before beginning the de-wax process, excess wax was removed by leaving the slides in an oven at $40{ }^{\circ} \mathrm{C}$ for $30 \mathrm{~min}$. Tissue samples were then de-waxed in xylene and rehydrated through an alcohol gradient to water and used for morphometry or immunohistochemistry.

\section{Morphometric evaluations}

Endometrial sections stained with anti-VEGFR2 serum in the immunohistochemistry study (see technique described below) were used for the morphometric study. Eleven pregnant mares on day 21, five on days 14 and 12 cyclic mares, six during the follicular phase, and six during the luteal phase were used. For data acquisition, the stratum spongiosum (including the stratum compactum) and the stratum basalis of dorsal endometrium from the posterior segment of both uterine horns in each animal were examined as separate layers. Pictures were taken at $200 \times$ magnification using a Nikon TE2000 microscope and captured using a Nikon DM1200F digital camera and the NIS-Elements AR 2.3 software was used to calculate all measurements. Three representative microscopic fields of the stratum spongiosum and stratum basalis were randomly selected for all measurements. Data represent the average of the three measurements at $200 \times$ magnification. The percentage of stromal area occupied by blood vessels, the percentage of endometrial area occupied by glands and stroma, and the number of uterine gland cross sections in each field of view were endpoints calculated. The average of data from three randomly selected fields of view in each location was used for the analysis.

\section{Immunohistochemistry}

After deparaffinization and rehydration, endometrial samples were submitted to antigen retrieval treatment to unmask antigenic sites by use of programmed heat and pressure. The slides were placed inside a receptacle containing $10 \mathrm{mM}$ sodium citrate buffer ( $\mathrm{pH}$ 6.0). This receptacle was placed inside a pressure chamber (Biocare Medical Decloaking Chamber, DC2002-CE; Biocare Medical, Concord, CA, USA) and the temperature programmed to $125^{\circ} \mathrm{C}$ for $30 \mathrm{~s}$ and then reduced to $90{ }^{\circ} \mathrm{C}$ for $10 \mathrm{~s}$ and allowed to cool at room temperature. When at room temperature, the slides were washed in tap water followed by TBS-Tween solution $(0.01 \mathrm{M}$ Tris-buffered saline, $0.15 \mathrm{M} \mathrm{NaCl}, 0.05 \%$ Tween 20, pH 7.4). Endogenous peroxidase was blocked by treating the slides by immersion in a solution of $3 \%$ hydrogen peroxidase in TBS for $15 \mathrm{~min}$ at room temperature followed by washing with TBSTween. Nonspecific antigen sites were blocked by incubation of the slides in a $10 \%$ goat serum in TBS-Tween solution for $30 \mathrm{~min}$ at room temperature. After blocking, the slides were incubated with the primary antibody. Four antisera were used and diluted as follows: an anti-VEGF (147) polyclonal rabbit 
serum, 1:200 (sc-507; Santa Cruz Biotechnology, Inc., Santa Cruz, CA, USA); an anti-VEGFR1 polyclonal rabbit serum, 1:200 (Flt-1 C-17: sc-316; Santa Cruz Biotechnology, Inc.); an anti-VEGFR2 monoclonal mouse serum, 1:200 (Flk-1 A-3: sc6251; Santa Cruz Biotechnology, Inc.); and an anti-Ki-67 polyclonal rabbit serum, 1:1500 (NCL-Ki67p; Novocastra Laboratories Ltd, Newcastle, UK). Slides were incubated with the primary antibodies for $45 \mathrm{~min}$ at room temperature and then washed with TBS-Tween. A universal probe capable of detecting rabbit and mouse antibodies followed by a polymer HRP was used to label the primary antibodies. For this step, the slides were incubated for $10 \mathrm{~min}$ at room temperature with the universal probe (Mach 4 universal AP polymer; M4U536G; Biocare Medical) and then for 10 more minutes with the HRP polymer (Mach 4 universal HRP polymer; M4U534G; Biocare Medical). The slides were washed with TBS-Tween and, for visualization of the binding sites, incubated with diaminobenzidine (K 047; Diagnostic BioSystems, Pleasanton, CA, USA) as substrate/chromogen for $4 \mathrm{~min}$ at room temperature, followed by TBS washing. Counterstaining was performed using hematoxylin for $3 \mathrm{~min}$ at room temperature followed by TBS and tap water washing. The endometrium sections were then labeled, dehydrated, and mounted in DPX resin. Negative and positive control slides were done for each run using the same described protocol. For negative controls, the primary antibody was replaced or by TBS-T solution or IGG in endometrium sections. For positive controls, horse kidney sections were used with the anti-VEGF and VEGF receptors sera and lymph node sections with the anti-Ki67 serum.

\section{RNA extraction and quantitative RT-PCR}

Total RNA was extracted from endometrial biopsies using the TRIZOL Plus RNA Purification Kit (Invitrogen Corp.). RNA samples (200 ng/reaction) were treated with RNase-free DNase (Applied Biosystems, Inc., Carlsbad, CA, USA) for $15 \mathrm{~min}$ at $37^{\circ} \mathrm{C}$, heat denatured $\left(75^{\circ} \mathrm{C}\right.$ for $\left.10 \mathrm{~min}\right)$, and then reverse transcribed using High Capacity cDNA Reverse Transcriptase Kit and random hexamers (Applied Biosystems). Real-time PCR was completed using SYBR Green PCR Master Mix (Applied Biosystems) and primers specific for equine VEGF (GenBank GenelD: DX010658), VEGFR1 (GenBank GenelD: AJ319908), VEGFR2 (GenBank GenelD: XM_001493036), or 185 GenBank Accession No. AJ311673) $\left(95^{\circ} \mathrm{C}\right.$ for $10 \mathrm{~min} ; 40$ cycles of $95{ }^{\circ} \mathrm{C}$ for $15 \mathrm{~s}, 55^{\circ} \mathrm{C}$ for $1 \mathrm{~min}, 72{ }^{\circ} \mathrm{C}$ for $1 \mathrm{~min} ; 55$ to $95^{\circ} \mathrm{C}$ Dissociation Event). Specificity of amplification was monitored by including non-reverse-transcribed RNA reactions for each sample and by completing a dissociation analysis at the end of each real-time run to verify the amplification of a single product. Changes in relative abundance of specific transcripts were examined using the ratio of the target RNA abundance relative to the reference standard using the following formula: $2^{-C_{\mathrm{T}} \text { (target) }} / 2^{-C_{\mathrm{T}}(18 \mathrm{~S})}$ (Michael et al. 2006).

\section{Statistical analyses}

Data were examined for normality with the KolmogorovSmirnov test. When the normality test was significant $(P<0.05)$, data were transformed to natural logarithms or square root to minimize heterogeneity of variance. Individual end points were analyzed for time effects (day), and comparisons involving groups were analyzed for main effects (group, time) and the interaction by least-squares ANOVA using the general linear model procedure of SAS (version 9.2; SAS Institute, Inc., Cary, NC, USA). Paired and unpaired Student's $t$-tests were used to detect differences between individual means within an end point and among groups within the same end point, when individual effects or an interaction was obtained. A probability of $P \leq 0.05$ indicated that a difference was significant. Data are presented as the mean \pm s.E.M.

\section{Declaration of interest}

The authors declare that there is no conflict of interest that could be perceived as prejudicing the impartiality of the research reported.

\section{Funding}

Projects P1-LS-07 and P2-LS-07. L A Silva was supported by a CAPES Scholarship (Brazil) to the University of Florida.

\section{Acknowledgements}

The authors thank personnel at the University of Florida Horse Unit for their assistance with pony care.

\section{References}

Albrecht ED, Aberdeen GW, Niklaus AL, Babischkin JS, Suresch DL \& Pepe G 2003 Acute temporal regulation of vascular endothelial growth/ permeability factor expression and endothelial morphology in the baboon endometrium by ovarian steroids. Journal of Clinical Endocrinology and Metabolism 88 2844-2852. (doi:10.1210/jc.2002-021546)

Allen WR, Gower S \& Wilsher S 2007 Immunohistochemical localization of vascular endothelial growth factor (VEGF) and its two receptors (Flt-I and $\mathrm{KDR}$ ) in the endometrium and placenta of the mare during the oestrous cycle and pregnancy. Reproduction in Domestic Animals 42 516-526. (doi:10.1111/j.1439-0531.2006.00815.x)

Amoroso EC 1952 Placentation. In Marshall's Physiology of Reproduction, vol 2, 3rd edn, pp 127-311. Ed. AS Parkes. London: Logmans Green and Co.

Bausero P, Cavaillé F, Méduri G, Freitas S \& Perrot-Applanat M 1998 Paracrine action of vascular endothelial growth factor in the human endometrium: production and target sites, and hormonal regulation. Angiogenesis 2 167-182. (doi:10.1023/A:1009292506879)

Betteridge KJ, Eaglesome MD, Mitchell D, Flood PF \& Beriault R 1982 Development of horse embryos up to twenty two days after ovulation: observations on fresh specimens. Journal of Anatomy 135 191-209.

Bourlev V, Volkov N, Pavlovitch S, Lets N, Larsson A \& Olovsson M 2006 The relationship between microvessel density, proliferative activity and expression of vascular endothelial growth factor-A and its receptors in eutopic endometrium and endometriotic lesions. Reproduction 132 501-509. (doi:10.1530/rep.1.01110)

Burton GJ, Watson AL, Hempstock J, Skepper JN \& Jauniaux E 2002 Uterine glands provide histiotrophic nutrition for the human fetus during the first trimester of pregnancy. Journal of Clinical Endocrinology and Metabolism 87 2954-2959. (doi:10.1210/jc.87.6.2954)

Burton GJ, Jauniaux E \& Charnock-Jones DS 2007 Human early placental development: potential roles of the endometrial glands. Placenta $\mathbf{2 8}$ (Suppl A) S64-S69. (doi:10.1016/j.placenta.2007.01.007)

Chenault JR 1980 Steroid metabolism by the early bovine conceptus - I. $5 \beta$ Reduction of neutral C19-steroids. Journal of Steroid Biochemistry 13 499-506. (doi:10.1016/0022-4731(80)90205-8) 
Chu JW, Sharom FJ, Oriol JG, Betteridge KJ, Cleaver BD \& Sharp DC 1997 Biochemical changes in the equine capsule following prostaglandininduced pregnancy failure. Molecular Reproduction and Development 46 286-295. (doi:10.1002/(SICl)1098-2795(199703)46:3<286::AIDMRD7 > 3.0.CO;2-L)

Cullinan-Bove K \& Koos RD 1993 Vascular endothelial growth factor/ vascular permeability factor expression in the rat uterus: rapid stimulation by estrogen correlates with estrogen-induced increases in uterine capillary permeability and growth. Endocrinology 133 829-837. (doi:10.1210/en.133.2.829)

Faber JJ \& Thornburg KL 1983 Placental Physiology. Structure and Function of Fetomaternal Exchange, New York: Raven.

Ferrara N 2004 Vascular endothelial growth factor: basic science and clinical progress. Endocrine Reviews 25 581-611. (doi:10.1210/er.2003-0027)

Ferrara N, Gerber H \& LeCouter J 2003 The biology of VEGF and its receptors. Nature Medicine 9 669-676. (doi:10.1038/nm0603-669)

Ford SP, Christenson RK \& Ford JJ 1982a Uterine blood flow and uterine arterial venous and luminal concentrations of estrogens on days 11, 13, and 15 after estrus in pregnant and nonpregnant sows. Journal of Reproduction and Fertility 64 185-190. (doi:10.1530/jrf.0.0640185)

Ford SP, Reynolds LP \& Magness RR $1982 b$ Blood flow to the uterine and ovarian vascular beds of gilts during the estrous cycle or early pregnancy. Biology of Reproduction 27 878-885. (doi:10.1095/biolreprod27.4.878)

Ginther OJ 1983 Fixation and orientation of the early equine conceptus. Theriogenology 19 613-623. (doi:10.1016/0093-691X(83)90181-4)

Ginther OJ 1984 Intrauterine movement of the early conceptus in barren and postpartum mares. Theriogenology 21 633-644. (doi:10.1016/0093691X(84)90448-5)

Ginther OJ 1992 Reproductive Biology of the Mare, 2nd edn. Cross Plains, WI: Equiservices Publishing.

Ginther OJ 1995 Ultrasonic Imaging and Animal Reproduction: Horses. Book 2. Cross Plains, WI: Equiservices Publishing.

Ginther OJ \& Silva LA 2006 Incidence and nature of disorientation of the embryo proper and spontaneous correction in mares. Journal of Equine Veterinary Science 26 249-256. (doi:10.1016/j.jevs.2006.04.001)

Girling JE \& Rogers PAW 2005 Recent advances in endometrial angiogenesis research. Angiogenesis 8 89-99. (doi:10.1007/s10456-005-9006-9)

Henneke DR, Potter GD, Kreider JL \& Yeates BF 1983 Relationship between condition score, physical measurements and body fat percentage in mares. Equine Veterinary Journal 15 371-372. (doi:10.1111/ j.2042-3306.1983.tb01826.x)

Hervé MAJ, Meduri G, Petit FG, Domet TS, Lazennec G, Mourah S \& Perrot-Applanat M 2006 Regulation of the vascular endothelial growth factor (VEGF) receptor Flk-1/KDR by estradiol through VEGF in uterus. Journal of Endocrinology 188 91-99. (doi:10.1677/joe.1.06184)

Heryanto B \& Rogers PAW 2002 Regulation of endometrial endothelial cell proliferation by oestrogen and progesterone in the ovariectomized mouse. Reproduction 123 107-113. (doi:10.1530/rep.0.1230107)

Jain R 2003 Molecular regulation of vessel maturation. Nature Medicine 9 685-693. (doi:10.1038/nm0603-685)

Kaczmarek MM, Waclawik A, Blitek A, Kowalczyk AE, Schams D \& Ziecik AJ 2008 Expression of the vascular endothelial growth factorreceptor system in the porcine endometrium throughout the estrous cycle and early pregnancy. Molecular Reprodution and Development $\mathbf{7 5}$ 362-372. (doi:10.1002/mrd.20815)

Klauber N, Rohan RM, Flynn E \& D'amato RJ 1997 Critical components of the female reproductive pathway are suppressed by the angiogenesis inhibitor AGM-1470. Nature Medicine 3 443-446. (doi:10.1038/ nm0497-443)

Lefranc AC \& Allen WR 2007 Endometrial gland surface density and hyperaemia of the endometrium during early pregnancy in the mare. Equine Veterinary Journal 39 511-515. (doi:10.2746/042516407X 235821)

Leith GS \& Ginther OJ 1984 Characterization of intrauterine mobility of the early equine conceptus. Theriogenology 22 401-408. (doi:10.1016/ 0093-691X(84)90460-6)

Lewis GS 1989 Prostaglandin secretion by the blastocyst. Journal of Reproduction and Fertility Supplement 37 261-267.

McDowell KJ, Sharp DC, Grubaugh W, Thatcher WW \& Wilcox CJ 1988 Restricted conceptus mobility results in failure of pregnancy maintenance in mares. Biology of Reproduction 39 340-348. (doi:10.1095/ biolreprod39.2.340)
Mendelsohn ME 2002 Genomic and nongenomic effects of estrogen in the vasculature. American Journal of Cardiology 90 (Suppl) 3F-6F. (doi:10. 1016/S0002-9149(02)02418-9)

Michael DD, Alvarez IM, Ocón OM, Powell AM, Talbot NC, Johnson SE \& Ealy AD 2006 Fibroblast growth factor-2 is expressed by the bovine uterus and stimulates interferon-tau production in bovine trophectoderm. Endocrinology 147 3571-3579. (doi:10.1210/en.2006-0234)

Raeside JI, Christie HL, Renaud RL, Waelchli RO \& Betteridge KJ 2004 Estrogen metabolism in the equine conceptus and endometrium during early pregnancy in relation to estrogen concentrations in yolk-sac fluid. Biology of Reproduction 71 1120-1127. (doi:10.1095/biolreprod.104. 028712)

Redmer DA, Aitken RP, Milne JS, Reynolds LP \& Wallace JM 2005 Influence of maternal nutrition on messenger RNA expression of placental angiogenic factors and their receptors at midgestation in adolescent sheep. Biology of Reproduction 72 1004-1009. (doi:10.1095/biolreprod.104.037234)

Reynolds LP \& Redmer DA 2001 Angiogenesis in the placenta. Biology of Reproduction 64 1033-1040. (doi:10.1095/biolreprod64.4.1033)

Reynolds LP, Borowicz PP, Vonnahme KA, Johnson ML, Grazul-Bilska AT, Redmer DA \& Caton JS 2005 Placental angiogenesis in sheep models of compromised pregnancy. Journal of Physiology 565 43-58. (doi:10. 1113/jphysiol.2004.081745)

Reynolds LP, Caton JS, Redmer DA, Grazul-Bilska AT, Vonnahme KA, Borowicz PP, Luther JC, Wallace JM, Wu G \& Spencer TE 2006 Evidence for altered placental blood flow and vascularity in compromised pregnancies. Journal of Physiology 572 51-58. (doi:10.1113/jphysiol. 2005.104430)

Risau W 1997 Mechanisms of angiogenesis. Nature 386 671-674. (doi:10. 1038/386671a0)

Rockwell LC, Pillai S, Olson CE \& Koos RD 2002 Inhibition of vascular endothelial growth factor/vascular permeability factor action blocks estrogen-induced uterine edema and implantation in rodents. Biology of Reproduction 67 1804-1810. (doi:10.1095/biolreprod.102.006700)

Roy H, Bhardwaj S \& Ylä-Herttuala S 2006 Biology of vascular endothelial growth factors. FEBS Letters $\mathbf{5 8 0}$ 2879-2887. (doi:10.1016/j.febslet. 2006.03.087)

Shemesh H, Milaguir F, Ayalon N \& Hansel W 1979 Steroidogenesis and prostaglandin synthesis by cultured bovine blastocysts. Journal of Reproduction and Fertility 56 181-185. (doi:10.1530/jrf.0.0560181)

Silva LA \& Ginther OJ 2006 An early endometrial vascular indicator of completed orientation of the embryo and the role of dorsal endometrial encroachment in mares. Biology of Reproduction 74 337-343. (doi:10. 1095/biolreprod.105.047621)

Silva LA \& Ginther OJ 2010 Local effect of the conceptus on uterine vascular perfusion during early pregnancy in heifers. Reproduction 139 453-463. (doi:10.1530/REP-09-0363)

Silva LA, Gastal EL, Beg MA \& Ginther OJ 2005 Changes in vascular perfusion of the endometrium in association with changes in location of the embryonic vesicle in mares. Biology of Reproduction 72 755-761. (doi:10.1095/biolreprod.104.036384)

Torry DS, Leavenworth J, Chang M, Maheshwari V, Groesch K, Ball ER \& Torry RJ 2007 Angiogenesis in implantation. Journal of Assisted Reproduction and Genetics 24 303-315. (doi:10.1007/s10815-0079152-7)

Yancopoulos GD, Davis S, Gale NW, Rudge JS, Wiegand AJ \& Holash J 2000 Vascular-specific growth factors and blood vessel formation. Nature 407 242-248. (doi:10.1038/35025215)

Zavy MT, Vernon MW, Sharp DC \& Bazer FW 1984 Endocrine aspects of early pregnancy in pony mares: a comparison of uterine luminal and peripheral plasma levels of steroids during the estrous cycle and early pregnancy. Endocrinology 115 214-219. (doi:10.1210/endo-115-1-214)

Zygmunt M, Herr F, Münsttedt K, Lang U \& Liang O 2003 Angiogenesis and vasculogenesis in pregnancy. European Journal of Obstetrics, Gynecology, and Reproductive Biology 110 S10-S18. (doi:10.1016/ S0301-2115(03)00168-4)

Received 3 May 2011

First decision 6 June 2011

Accepted 7 July 2011 